\title{
L-S-HOMOTOPY CLASSES ON THE TOPOLOGICAL IMAGE OF A PROJECTIVE PLANE
}

\author{
MARSTON MORSE
}

1. Introduction. Models for the L-S-(locally simple) homotopy classes of closed $p$-curves ( $p=$ parameterized) on any 2-manifold $S$ have been announced in Morse [1]. ${ }^{1}$ Proofs have been given only for the case in which $S$ is orientable. The present paper will treat the case in which $S$ is the top. (topological) image of a projective plane. The proofs in the case of a general non-orientable surface can be given by an appropriate modification of methods of Morse [1] and of the present paper.

Recall that one writes $f \approx 0$ when $f$ is a closed $p$-curve homotop. to zero. Deferring technical definitions until later sections, we can state the principal theorem as follows.

TheOREM 1.1. Let $h$ be a simple closed p-curve on the top. image $S$ of a projective plane with $h$ not $\approx 0$ on $S$. Let $h^{(n)}(n>0)$ be a closed p-curve on $S$ which traces $h n$ times. Any L-S-closed p-curve $f$ on $S$ is in the L-Shomotopy class of $h^{(1)}$ or $h^{(3)}$ if $h$ not $\approx 0$, and of $h^{(2)}$ or $h^{(4)}$ if $h \approx 0$. No two of the p-curves $h^{(1)}, h^{(2)}, h^{(3)}, h^{(4)}$ are in the same L-S-homotopy class.

For theorems on regular closed curves in the plane see Whitney, and H. Hopf. For L-S-closed curves in the plane see Morse [2] and Morse and Heins [1]. For a use of L-S-curves in studying deformation classes of meromorphic functions see Morse and Heins [2].

2. L-S-curves and deformations. Let $C$ represent the unit circle on which $|z|=1$ in the plane of the complex variable $z=u+i v$. With $z=e^{i \theta}$ on $C$, we assign $C$ the sense of increasing $\theta$. Let $S$ be an arbitrary 2-manifold. A closed p-curve on $S$ is a continuous mapping $f$ of $C$ into $S$ such that the image of $z$ in $C$ is a point $f(z)$ in $S$. Two $p$-curves $f_{1}$ and $f_{2}$ are regarded as the same if and only if

$$
f_{1}(z)=f_{2}(z)
$$

for every $z$ in $C$. The union of the points $f(z)$ in $S$ as $z$ ranges over $C$ is called the carrier of $f$. The simplest case arises when the points $f(z)$ are in 1-1 correspondence with their antecedents $z$ in $C$, and in this case $f$ is termed simple.

Let $f$ be a continuous mapping of $C$ into $S$. Let $\lambda$ be any sense

Received by the editors August 13, 1948.

${ }^{1}$ Numbers in brackets refer to the references cited at the end of the paper. 
preserving top. (that is, 1-1 and continuous) mapping of $C$ onto $C$ with $\lambda(z)$ the image in $S$ of $z$ in $C$. Then $f$ and $f \lambda$ are termed equivalent mappings of $C$ into $S$ or equivalent $p$-curves on $S$. Here $f \lambda$ symbolizes the function whose value at $z$ is $f[\lambda(z)]$. In earlier papers the class of mappings equivalent to a given mapping has been termed a curve, as distinguished from a $p$-curve. We shall here find it simpler to rely on $p$-curves and make use of Lemma 2.1, according to which any two equivalent closed $p$-curves are in the same L-S-homotopy class.

A closed $p$-curve $f$ on $S$ will be termed L-S if there is a positive constant $e$ so small that the mapping under $f$ of any arc of $C$ with length less than $e$ is top. Hence there exists a constant $e_{1}>0$ so small that any subarc of $f$ whose carrier has a diameter less than $e_{1}$ is simple. Such a constant $e_{1}$ is called a norm of local simplicity of $f$.

The L-S-homotopy class $[f]$. Let $J$ be the interval $0 \leqq t \leqq 1, t$ the "time." A deformation of $f$ on $S$ is a continuous mapping $D$ of $C \times J$ into $S$ such that the image of a point $(z, t)$ in $C \times J$ is a point $D(z, t)$ in $S$, with

$$
D(z, 0)=f(z)
$$

initially (that is, when $t=0$ ). For $t$ fixed in $J, D$ defines a mapping ${ }^{2}$ $D(\cdot, t)$ of $C$ into $S$, and thus a p-curve $f^{t}$ termed the deform of $f$ at the time $t$. We say that $f$ is deformed through the family $f^{t}$ into $f^{1}$. If the deforms $f^{t}, 0 \leqq t \leqq 1$, possess a common norm of local simplicity, $D$ will be said to be L-S. The class of $p$-curves $f^{1}$ into which $f$ can be L-S-deformed on $S$ is termed the L-S-homotopy class $[f]$ of $f$ on $S$. By virtue of a proof similar to that of Lemma 28.1 of Morse [2] we can affirm the following.

Lemma 2.1. Any two equivalent closed p-curves on $S$ are in the same L-S-homotopy class on $S$.

A first objective of this paper is the proof of Theorem 1.1. Models for the L-S-homotopy class $[f]$ on the top. image $S$ of a projective plane will be determined as indicated. In case the given $p$-curve $f \approx 0$, the $M$-order of $f$, as defined in Morse [1, $\$ 4]$, is 1 , or $2 \bmod 2$, according as $[f]=\left[h^{(2)}\right]$ or $[f]=\left[h^{(4)}\right]$. Here $M$ is the top. sphere covering $S$.

In case the given $p$-curve $f$ not $\approx 0$, a new $S$-difference order $d_{S}(f)$ is defined in $\S 7$ and $[f]=\left[h^{(1)}\right]$ or $\left[h^{(3)}\right]$ according as

$$
d_{S}(f)=1 \text { or } 3
$$

The value of $d_{S}(f)$ will be shown to be independent of $f$ in its L-S-

\footnotetext{
${ }^{2}$ For fixed $t, D(\cdot, t)$ symbolizes the function whose value at $z$ in $C$ is $D(z, t)$.
} 
homotopy class, and to be a top. invariant in the following sense. If $S^{\prime}$ is a top. image of $S$ with $f^{\prime}$ on $S^{\prime}$ the top. image of $f$ on $S$, then

$$
d_{S}(f)=d_{S^{\prime}}\left(f^{\prime}\right) \quad(\bmod 4) .
$$

An important special result in the case $f$ not $\approx 0$ is that $d_{S}(f)=1 \bmod 4$ if and only if $[f]$ contains a simple closed $p$-curve.

3. The 2-sphere $M$ covering $\Pi$. We shall begin with a special model II of a projective plane obtained by identifying diametrically opposite points

$$
x=\left(x_{1}, x_{2}, x_{3}\right), \quad-x=\left(-x_{1},-x_{2},-x_{3}\right)
$$

of a 2-sphere

$$
M: \quad x_{1}^{2}+\stackrel{x_{2}^{2}}{x^{2}} x_{3}^{2}=1
$$

A point in $I I$ can accordingly be given by a pair $(x,-x)$ of diametrically opposite points $x$ and $-x$ in $M$. We understand that the point $(x,-x)$ in $\Pi$ equals the point $(-x, x)$ of $\Pi$. We say that $x$ in $M$ covers the point $(x,-x)=(-x, x)$ in $\Pi$, and denote this point in $\Pi$ by $A(x)$. We say also that $x$ on $M$ projects into $A(x)$ on $\Pi$.

The mapping $A$ of $M$ onto II has two fundamental properties: (1) for $x$ and $y$ in $M$

$$
A(x)=A(y)
$$

if and only if $x= \pm y$; (2) the mapping $A$ is locally top.

Let $\phi$ be any mapping of an abstract set $E$ into $M$. Then ${ }^{3} A \phi$ is a mapping of $E$ into $\Pi$ termed the $A$-projection of $\phi$ into $\Pi$.

A closed $p$-curve $F$ mapping $C$ into $M$ will be termed $R$-invariant (reflection invariant) if for every $z$ in $C$

$$
F(-z)=-F(z) \text {. }
$$

The mapping $\mu$ of $C$ onto the semi-circle $C_{1}$. We shall make frequent use of a mapping $\mu$ of $C$ onto the semi-circle

$$
C_{1}: \quad z=e^{\theta i},
$$

$$
0 \leqq \theta<\pi
$$

of $C$. Explicitly, with $z=e^{i \theta}$, set

$$
\mu(z)=e^{i(\theta / 2)} \quad(0 \leqq \theta<2 \pi) .
$$

The mapping $\mu$ of $C$ into $C_{1}$ is continuous, except at $z=1$. Let $C_{2}$ be the residual semi-circle $C-C_{1}$ of $C$. When $F$ is $R$-invariant the

${ }^{3}$ Here and elsewhere a functional operation such as $A$ on $\phi$ is indicated by $A \phi$. The value of $A \phi$ at a point $x$ of $E$ will be denoted by $A \phi(x)$ and not by $A[\phi(x)]$. 
mapping $F \mu$ of $C$ into $M$ determines the mapping $F$ of $C$ into $M$ in accordance with the equations

$$
\begin{aligned}
& F \mu\left(z^{2}\right)=F(z) \\
& {\left[z \in C_{1}\right] \text {, }} \\
& F \mu\left(z^{2}\right)=-F(z) \\
& {\left[z \in C_{2}\right] \text {. }}
\end{aligned}
$$

By definition $\mu$ is single-valued; it is one branch of $z^{1 / 2}$ on $C$, and to avoid confusion is not to be continued into the other branch. The mapping $F \mu$ of $C$ into $M$ is discontinuous at the point $z=1$ of $C$. In fact,

$$
F_{\mu}(1)=-F_{\mu}\left(1^{-}\right)
$$

where $F \mu\left(1^{-}\right)$indicates the limit of $F \mu(z)$ as $z$ tends to $z=1$ on $C$ from the arc of $C$ on which $\pi<\operatorname{arc} z<2 \pi$.

When $F$ is an $R$-invariant closed $p$-curve on $M$, the mapping

$$
f^{F}=A F \mu
$$

of $C$ into $\Pi$ is a closed $p$-curve on $\Pi$ by virtue of (3.7). The $p$-curve $f^{F}$ is the $A$-projection of $F \mu$ on $\Pi$.

A deformation $\Delta$ of a closed $p$-curve $F$ on $M$ is a continuous mapping of $C \times J$ into $M$ in which the image of a point $(z, t)$ of $C \times J$ is a point $\Delta(z, t)$ in $M$, with

$$
\Delta(z, 0)=F(z) \quad[z \in C]
$$

initially, that is, for $t=0$. Such a deformation is termed $R$-invariant if

$$
\Delta(-z, t)=-\Delta(z, t)
$$

for each point $(z, t)$ in $C \times J$. Suppose then that $\Delta$ is $R$-invariant. Then $F$ is necessarily $R$-invariant. The deform $F^{t}$ of $F$ under $\Delta$ is the $R$-invariant mapping $\Delta(\cdot, t)$ of $C$ into $M$. The $A$-projection $A F \mu$ of $F \mu$ is a closed $p$-curve $f^{F}$ on $\Pi$, and $A \Delta \mu$ is a deformation of $f^{F}$ on II. We draw the following conclusion:

Lemma 3.1. If an $R$-invariant $L$-S-closed p-curve $F$ on $M$ admits an $R$-invariant $L$-S-deformation on $M$ through p-curves $F^{t}$, then the $A$ projection on $\Pi$ of $F \mu$ admits a L-S-deformation on $\Pi$ through the closed p-curves $A F^{t} \mu$ on $\Pi$.

4. The cases $f \approx 0$ and $f$ not $\approx 0$. Let $f$ be a closed $p$-curve on $\Pi$. Let $x_{0}$ and $-x_{0}$ be the points in $M$ which cover $f(1)$. Let $a$ be either one of the points $x_{0},-x_{0}$. Given $f$ and $a$ it follows from the local top. character of the projection $A$ of $M$ onto $\Pi$ that there exists a unique continuous mapping of $\phi_{a}^{f}$ of the $\theta$-axis into $M$ such that (with $\left.z=e^{i \theta}\right)$ 


$$
\phi_{a}^{f}(0)=a \quad A \phi_{a}^{f}(\theta)=f(z) \quad(-\infty<\theta<+\infty) .
$$

It follows from (4.1) that

$$
A \phi_{a}^{f}(\theta+2 \pi)=A \phi_{a}^{f}(\theta) .
$$

Recall that for points $x$ and $y$ in $M, A(x)=A(y)$ if and only if $x= \pm y$. From (4.2) then

$$
\phi_{a}^{f}(\theta+2 \pi)= \pm \phi_{a}^{f}(\theta) .
$$

The sign in (4.3) is independent of $\theta$ and is + if and only if $f \approx 0$ in accordance with the following lemma.

Lemma 4.1. If $f$ is a closed p-curve on $\Pi$ and a a point in $M$ such that $A(a)=f(1)$, and if $\phi_{a}^{f}$ is the unique continuous mapping of the $\theta$-axis into $M$ such that (4.1) holds given a, then a necessary and sufficient condition that

$$
\phi_{a}^{f}(\theta+2 \pi)=\phi_{a}^{f}(\theta) \quad(-\infty<\theta<+\infty)
$$

is that $f \approx 0$ on $\Pi$.

If (4.4) holds the equation

$$
F_{a}^{f}(z)=\phi_{a}^{f}(\theta)
$$$$
\left(z=e^{i \theta}\right)
$$

defines a single-valued mapping $F_{a}^{f}$ of $C$ into $M$. Thus $F_{a}^{f}$ is a closed $p$-curve on $M$, and as such is deformable on $M$ through a continuous family $F_{a}^{f t}, 0 \leqq t \leqq 1$, of closed $p$-curves on $M$ into a $p$-curve, whose carrier is a point of $M$. The $A$-projections $A F_{a}^{f t}$ into $\Pi$ of these $p$ curves deform $f$ on $\Pi$ into a $p$-curve on $\Pi$ whose carrier is a point. Hence $f \approx 0$ on II if (4.4) holds.

Conversely suppose that $f \approx 0$ on $\Pi$, or more specifically that $f$ on $\Pi$ is deformed into a $p$-curve whose carrier is a point, through a family $f^{t}(0 \leqq t \leqq 1)$ of closed $p$-curves on $\Pi$. With $\phi_{a}^{f}$ given in terms of $f$ by (4.1) a continuous mapping $\phi_{a}^{f t}$ of the $\theta$-axis into $M$ can be determined by continuation with respect to increasing $t$, with

$$
\phi_{a}^{f 0}(\theta) \equiv \phi_{a}^{f}(\theta)
$$

initially, and

$$
A \phi_{a}^{f t}(\theta) \equiv f^{t}(z) \quad\left[\text { for } z=e^{i \theta}\right]
$$

where $\phi_{a}^{f t}(\theta)$ is continuous in $(\theta, t)$ for $0 \leqq t \leqq 1$ and arbitrary $\theta$.

As a consequence of (4.6) 


$$
\phi_{a}^{f t}(2 \pi)= \pm \phi_{a}^{f t}(0)
$$

where the sign in (4.7) is independent of $t$ on $[0,1]$. For $t$ sufficiently near 1 on $[0,1]$ the + sign must hold in $(4.7)$ since the carrier of $f^{1}$ in (4.6) is a point and $A$ is locally top. Hence the + sign must hold in (4.7) when $t=0$ as well. Thus (4.4) holds when $f \approx 0$ on II.

Antecedents and $\mu$-antecedents on $M$ of p-curves on $\Pi$. If $f$ is a closed $p$-curve on $\Pi$, any closed $p$-curve $F$ on $M$ such that $A F=f$ will be called an antecedent on $M$ to $f$ on II. If $f$ not $\approx 0$ no closed $p$-curve $F$ on $M$ can be antecedent to $f$, because (4.4) cannot hold in this case. However we shall verify the following. When $f$ not $\approx 0$ there always exists (Lemma 4.2) an $R$-invariant closed $p$-curve $F$ on $M$ such that $A F \mu=f$. Such a closed $p$-curve $F$ will be called a $\mu$-antecedent of $f$.

LeMma 4.2. Let $f$ be a closed p-curve on $\Pi$. If $f \approx 0$ on $\Pi$ there exist just two closed p-curves $F^{f}$ antecedent on $M$ to $f$ on $\Pi$. If $f$ not $\approx 0$ there exist just two closed p-curves $F^{f}, \mu$-antecedent on $M$ to $f$ on $\Pi$.

The two antecedents ( $\mu$-antecedents) $F$ and $F^{*}$ of $f$ satisfy the relation $F(z)=-F^{*}(z)$.

Case I. $f \approx 0$. We start with $\phi_{a}^{f}$ as defined in (4.1), with $a=x_{0}$ or $-x_{0}$ where $A\left(x_{0}\right)=f(1)$. Let $F_{a}^{f}$ then be defined as in (4.5). In case I, (4.4) holds, so that $F_{a}^{f}$ is closed on $M$. The $A$-projection of $F_{a}^{f}$ is $f$ in accordance with (4.1). There are accordingly at least two closed $p$-curves $F_{a}^{f}\left(a=x_{0}\right.$ or $\left.-x_{0}\right)$ antecedent to $f$ on $\Pi$.

Any other closed $p$-curve $F$ on $M$ such that $A F=f$ must satisfy the condition

$$
F(1)=a \quad\left(a=x_{0} \text { or }-x_{0}\right) .
$$

By virtue of the continuity of the mapping $F^{f}$ and the top. character of $A, F$ is thereby uniquely determined by $f$ and $a$, and so must equal $F_{a}^{\prime}$. There are accordingly just two closed $p$-curves $F$ antecedent on $M$ to $f$ on $\Pi$ when $f \approx 0$.

Case II. $f$ not $\approx 0$. We start again with $\phi_{a}^{f}$ as defined in (4.1). In Case II we define $F_{a}^{f}$ as a $p$-curve on $M$ such that for $w=e^{i \alpha}$

$$
F_{a}^{f}(w)=\phi_{a}^{f}(2 \alpha) \quad[-\infty<\alpha<\infty] .
$$

That $F_{a}^{f}$ is single-valued for each $w$ in $C$, and $R$-invariant, follows from the relation

$$
\phi_{a}^{f}(\alpha+2 \pi)=-\phi_{a}^{f}(\alpha)
$$

which holds by virtue of Lemma 4.1 and (4.3). In fact 


$$
F_{a}^{f}(-w)=\phi_{a}^{f}(2[\alpha+\pi])=-\phi_{a}^{f}(2 \alpha)=-F_{a}^{f}(w)
$$

for every $w$ in $C$. Relation (4.8) implies that

$$
F_{a \mu}^{f}(z)=\phi_{a}^{f}(\theta) \quad\left\lfloor z=e^{i \theta}, 0 \leqq \theta<2 \pi\right\rfloor
$$

and it follows from (4.1) that $A F_{a}^{\gamma} \mu=f$. While $F_{a}^{\dagger} \mu$ is discontinuous at $z=1$ on account of (4.9), $A F_{a}^{f} \mu$ is continuous at $z=1$. In Case II there accordingly exists two closed $p$-curves $F_{a}^{f}\left(a= \pm x_{0}\right), \mu$-antecedent on $M$ to $f$ on $\Pi$.

Any other closed $p$-curve $F$ on $M$ such that $A F \mu=f$ must satisfy the condition $F(1)=a,\left[a= \pm x_{0}\right]$ and by a process of continuation be uniquely determined by the relation $A F \mu=f$ as the $R$-invariant closed $p$-curve $F_{a}^{f}$. The $p$-curves $F_{a}^{s}\left[a= \pm x_{0}\right]$ are accordingly the only closed $p$-curves $\mu$-antecedent on $M$ to $f$ on $\Pi$ when $f$ not $\approx 0$.

The preceding lemma can be extended to deformations $D$ on $M$ as follows.

Lemma 4.3. Corresponding to any continuous deformation $D$ on $\Pi$ of a closed p-curve $f$ such that $f \approx 0[f$ not $\approx 0]$ on $\Pi$, and to either of the two antecedents [ $\mu$-antecedents] $F^{f}$ of $f$, there exists a unique deformation $\Delta$ of $F^{f}$ on $M$ such that $A \Delta=D$ when $f \approx 0$, while $\Delta$ is $R$-invariant and $A \Delta \mu=D$ when $f$ not $\approx 0$.

We term the deformation $\Delta$ of the lemma antecedent on $M$ to $D$ on $\Pi$ when $A \Delta=D$, and $\mu$-antecedent when $A \Delta \mu=D$.

The proof of Lemma 4.3 is so similar to that of Lemma 4.2 that it need only be indicated.

Let $f^{t}, 0 \leqq t \leqq 1$, be the deform of $f$ under $D$ at the time $t$. Let $X_{0}(t)$ and $-X_{0}(t)$ be the two points in $M$ which cover $f^{t}(1)$ on $\Pi$, $X_{0}$ being chosen so that it is continuous for $t$ in $[0,1]$. Let $\Theta$ represent the $\theta$-axis. Let $a$ be either of the functions $\pm X_{0}$. There exists a unique continuous mapping $\Phi_{a}$ of $\Theta \times J$ into $M$ such that (with $\left.z=e^{i \theta}\right)$

$$
\Phi_{a}(0, t)=a(t), \quad A \Phi_{a}(\theta, t)=D(z, t)
$$

for each point $(\theta, t)$ on $\Theta \times J$. Equation (4.11) replaces (4.1) while the analogue of $(4.3)$ is

$$
\Phi_{a}(\theta+2 \pi, t)= \pm \Phi_{a}(\theta, t)
$$

where the sign is + if and only if $f \approx 0$ on $\Pi$.

Case I. $f \approx 0$. In this case the required deformation $\Delta$ is defined by the equation 


$$
\Delta_{a}(z, t)=\Phi_{a}(\theta, t)
$$$$
\left[z=e^{i \theta}\right] \text {. }
$$

Then $A \Delta_{a}=D$ in accordance with (4.11).

Case II. $f$ not $\approx 0$. One here sets

$$
\Delta_{a}(w, t)=\Phi_{a}(2 \alpha, t)
$$$$
\left[w=e^{i \alpha}\right]
$$

and observes that $\Delta_{a}$ is single-valued and $R$-invariant by virtue of (4.12), the sign - prevailing in (4.12). Finally (4.13) implies that

$$
\Delta_{a}[\mu(z), t]=\Phi_{a}(\theta, t) \quad\left(z=e^{i \theta}, 0 \leqq \theta<2 \pi\right)
$$

for each $t$ on $[0,1]$, so that $A \Delta_{a} \mu=D$.

In either case the uniqueness of a deformation $\Delta$ satisfying the lemma when an antecedent ( $\mu$-antecedent) $F^{t}$ of $f$ is given, follows as in the proof of Lemma 4.3.

If $F$ is an $R$-invariant closed $p$-curve on $M$ the class of all $R$-invariant closed $p$-curves which admit $R$-invariant L-S-deformations into $F$ on $M$ will be called an $R$-invariant $L$-S-homotopy class on $M$.

The following theorem reduces the problem of determining the L-S-homotopy classes on $I I$ to a problem on $M$. It is a consequence of the preceding lemmas including Lemma 3.1.

THEOREM 4.1. A necessary and sufficient condition that L-S-closed p-curves $f_{1}$ and $f_{2} \approx 0[$ not $\approx 0]$ on $\Pi$ be in the same L-S-homotopy class on $\Pi$ is that an antecedent [ $\mu$-antecedent] $F^{f_{1}}$ and $F_{f_{2}}$ of $f_{1}$ and $f_{2}$ respectively be in the same L-S-homotopy class [R-invariant L-S-homotopy class] on $M$.

In case $f \approx 0$ on $\Pi$ models for the L-S-homotopy classes of $f$ can accordingly be inferred from those on the 2 -sphere $M$. Such models on $M$ are given in Theorem 4.2 of Morse [1].

The model $p$-curve $k$ on $\Pi$, and $\Gamma$ on $M$. We shall introduce a simple closed $p$-curve $k$ on $\Pi$, with carrier on $\Pi$ covered by a great semicircle on $M$. More definitely we suppose that $k$ has $a \mu$-antecedent $\Gamma$ on $M$ given by the mapping

$$
x_{1}+i x_{2}=z, \quad x_{3}=0 \quad[z \in C],
$$

of the circle $C$ into $M$. For each integer $n>0$ let closed p-curves $k^{(n)}$ on $\Pi$ and $\Gamma^{(n)}$ on $M$ be defined by the equations

$$
k^{(n)}(z)=k\left(z^{n}\right), \quad \Gamma^{(n)}(z)=\Gamma\left(z^{n}\right) \quad[z \in C] .
$$

The p-curves $k^{(1)}$ and $k^{(3)}$ have $\Gamma$ and $\Gamma^{(3)}$ as $\mu$-antecedents on $M$, while $k^{(2)}$ and $k^{(4)}$ have $\Gamma$ and $\Gamma^{(2)}$ as antecedents on $M$. Theorem 4.2 of Morse [1] gives the following. 
Theorem 4.2. Any L-S-closed p-curve $f \approx 0$ on $\Pi$ is in the $L$-Shomotopy class of $k^{(2)}$ or $k^{(4)}$ on $\Pi$, while $\left[k^{(2)}\right] \neq\left[k^{(4)}\right]$ on $\Pi$.

That $\left[k^{(2)}\right] \neq\left[k^{(4)}\right]$ on $\Pi$ follows from the fact that $[\Gamma] \neq\left[\Gamma^{(2)}\right]$ on $M$. For the equality $\left[k^{(2)}\right]=\left[k^{(4)}\right]$ on $\Pi$ would imply that $[\Gamma]=\left[\Gamma^{(2)}\right]$ on $M$ by virtue of Theorem 4.1 .

Given $f \approx 0$ on $I I$ the problem of determining to which of the two homotopy classes, $\left[k^{(2)}\right]$ or $\left[k^{(4)}\right], f$ belongs is equivalent to the problem of determining to which of the two homotopy classes, $[\Gamma]$ or $\left[\Gamma^{(2)}\right]$ on $M$, an antecedent $F$ of $f$ belongs on $M$. This problem is resolved by the determination of the $M$-order $p(F)$, of $F$, as shown in $\S 4$ of Morse [1]. In fact

$$
[F]=[\Gamma] \text { or }\left[\Gamma^{(2)}\right]
$$

according as $p(F)=1$ or $2 \bmod 2$. As shown in Morse [1], $p(F)$ is a topological invariant of $M$ and $F$, and in particular is invariant under any " $R$-invariant" homeomorphism $T$ of $M$, that is, one for which

$$
T(-x)=-T(x) \quad(x \in M),
$$

and is accordingly a top. invariant of $\Pi$. Finally if $F$ and $F^{\prime}$ are L-S-closed $p$-curves on $M$

$$
p(F)=p\left(F^{\prime}\right)
$$

if and only if $[F]=\left[F^{\prime}\right]$ on $M$, and accordingly if and only if $[A F]$ $=\left[A F^{\prime}\right]$ on $\Pi$.

We turn accordingly to the case $f$ not $\approx 0$ on $\Pi$.

5. L-S-homotopy classes when $f$ not $\approx 0$ on $I$. Let $F$ be an $R$-invariant L-S-closed $p$-curve on $M$. In accordance with Theorem 4.1 we seek a model for the $R$-invariant L-S-homotopy classes of $F$ on $M$. To that end we refer to the semi-circle $C_{1}$ defined by $z=e^{\theta i}$ for $0 \leqq \theta<\pi$, and to the residual semi-circle $C_{2}$ defined by $z=e^{\theta i}$ when $\pi \leqq \theta<2 \pi$. Let $F_{1}$ and $F_{2}$ be submappings of $F$ defined by the equations (a superimposed bar indicates closure)

$$
\begin{array}{lr}
F_{1}(z)=F(z) & {\left[z \in \bar{C}_{1}\right],} \\
F_{2}(z)=F(z) & {\left[z \in \bar{C}_{2}\right]}
\end{array}
$$

and term $F_{1}$ the kernel of $F_{1}$ and $F_{2}$ the kernel residue. We shall be concerned with various continuous mappings of $\bar{C}_{1}$ into $M$ and will term such mappings $p$-arcs on $M$.

Various elementary $p$-arcs and $p$-curves on $M$ will be defined and analyzed for later use. In defining kernels $F_{1}$ the path which $F(z)$ traces as $z$ traces $\bar{C}_{1}$ will be given. These paths will be ordered finite 
sequences of simple, sensed arcs successively joined to form a continuous curve. The paths used will be rectifiable. From a path $\alpha$ a kernel $F_{1}=\{\alpha\}$ will be formed by making $z$ in $\bar{C}_{1}$ correspond to that point $F_{1}(z)$ in $\alpha$ which divides $\alpha$ in the same ratio with respect to arc length as that in which $z$ divides $\bar{C}_{1}$ with respect to arc length. The kernel residue $F_{2}$ will be defined by setting

$$
F_{2}(-z)=-F_{1}(z) \quad\left[z \in \bar{C}_{1}\right] .
$$

In order that $F$ so defined be L-S it is sufficient that $F_{1}$ be L-S and that the images on $M$ under $F$ of sufficiently small neighborhoods of $z=1$ in $C$ be simple.

A symbolism is needed for a path $\alpha$ which is a product

$$
\alpha=a_{1} a_{2} \cdots a_{n}
$$

of simple, regular, sensed, closed curves $a_{1}, \cdots, a_{n}$, with $a_{k}$ positively tangent to $a_{k+1}(k=1, \cdots, n-1)$ at a prescribed point $P_{k}$. If $a$ is a simple, sensed arc or closed curve, and $P$ and $Q$ are two points in $a$, $a(P, Q)$ shall denote the subarc (if any exists) of $a$ leading from $P$ to $Q$ on $a$. With this understood $\alpha$ shall denote the path defined by the sequence of simple arcs (with $a_{1}\left(P_{1}, P_{1}\right)$ the arc $a_{1}$ cut at $P_{1}$ ),

$$
\begin{gathered}
a_{1}\left(P_{1}, P_{1}\right) a_{2}\left(P_{1}, P_{2}\right) \cdots a_{n-1}\left(P_{n-2}, P_{n-1}\right), \\
a_{n}\left(P_{n-1}, P_{n-1}\right) a_{n-1}\left(P_{n-1}, P_{n-2}\right) \cdots a_{2}\left(P_{2}, P_{1}\right) .
\end{gathered}
$$

We admit the possibility that $a_{1}$ is not a closed curve, but rather the closure of a simple arc, while $a_{2} \cdots a_{n}$ remain simple closed curves. In such a case $P_{1}$ is to be an inner point of $a_{1}$. If $P$ and $P^{\prime}$ are the initial and final points of $a_{1}$, the preceding sequence (5.1) is to be altered by replacing $a_{1}\left(P_{1}, P_{1}\right)$ by $a_{1}\left(P, P_{1}\right)$ and $a_{1}\left(P_{1}, P^{\prime}\right)$ is to be added to the sequence.

The elementary $p$-arcs on $M$ to be used in defining model kernels $F_{1}$ on $M$ can now be defined. Let $\gamma$ be the simple arc

$$
x_{1}=\cos \theta, \quad x_{2}=\sin \theta, \quad x_{3}=0 \quad(0 \leqq \theta<\pi)
$$

taken in the sense of increasing $\theta$. Let $\lambda$ be a small sensed circle of diameter $<1$, with $x_{3} \geqq 0$ thereon, positively tangent to $\gamma$ at the mid point $(0,1,0)$ of $\gamma$. Let $\lambda^{-1}$ be the reflection of $\lambda$ in the plane $\left[x_{3}=0\right]$. For $n$ a positive integer $\lambda^{n}$ shall formally symbolize $\lambda \cdots \lambda$ with $n$ factors $\lambda$, while $\lambda^{-n}$ shall formally symbolize $\lambda^{-1} \cdots \lambda^{-1}$ with $n$ factors $\lambda^{-1}$. Let $q$ be any nonvanishing integer. We introduce a product path $\gamma \lambda^{q}$ in which $(0,1,0)$ is the point of contact of successive factors. Then $\left\{\gamma \lambda^{q}\right\}$ is a well-defined $p$-arc on $M$ which, taken 
as a kernel $F_{1}$, leads to a L-S-closed $p$-curve $F$ on $M$. We shall prove the following lemma.

LEMMA 5.1. The p-arc $\left\{\gamma \lambda^{q}\right\}$ admits a L-S-deformation on $M$ in which sufficiently short initial and final subarcs of $\gamma$ remain simple with invariant carriers, and in which $\left\{\gamma \lambda^{q}\right\}$ is deformed into $\{\gamma\}$ when $q$ is even, and into $\{\gamma \lambda\}$ when $q$ is odd.

For simplicity we begin with $\gamma \lambda^{2}$. The circle $\lambda$ can be deformed on $M$ through circles tangent to $\gamma$ at $(0,1,0)$ into $\lambda^{-1}$, so that $\left\{\gamma \lambda^{2}\right\}$ is L-S-deformable on $M$ into $\left\{\gamma \lambda \lambda^{-1}\right\}$. The point of contact of $\lambda^{-1}$ with $\lambda$ can be continuously regressed on $\lambda$ to the point of maximum $x_{3}$ on $\lambda$, varying $\lambda^{-1}$ through circles $\lambda_{t}^{-1}, 0 \leqq t \leqq 1$, of fixed radius. Then $\lambda_{1}^{-1}$ is the terminal circle in this deformation of $\lambda^{-1}$. Observe that $\lambda \lambda_{1}^{-1}$ is a figure eight with $x_{3}>0$ thereon, except at the point of contact of $\lambda$ with $\gamma$ at $(0,1,0)$. It is clear that $\left\{\gamma \lambda \lambda_{1}^{-1}\right\}$ is L-S-deformable into $\{\gamma\}$, and that the whole deformation of $\left\{\gamma \lambda^{2}\right\}$ into $\{\gamma\}$ can be so made that sufficiently short initial and final arcs of $\gamma$ remain simple with invariant carriers.

In the same way, it is clear that for $q>2\left[\gamma \lambda^{q}\right\}$ is L-S-deformable successively into

$$
\left\{\gamma \lambda^{q-2} \lambda \lambda_{1}^{-1}\right\} \quad\left\{\gamma \lambda^{q-2}\right\}
$$

so that an induction with respect to $q$ shows that the lemma is true if $q>2$. A reflection in the plane at $x_{3}=0$ makes it appear that for $q<0,\left\{\gamma \lambda^{q}\right\}$ is L-S-deformable in the required manner into $\{\gamma\}$ when $q$ is even, and into $\left\{\gamma \lambda^{-1}\right\}$, when $q$ is odd. But the above deformation of $\lambda$ into $\lambda^{-1}$ shows that $\left\{\gamma \lambda^{-1}\right\}$ is L-S-deformable into $\{\gamma \lambda\}$, and the proof of the lemma is complete.

The succeeding proofs will be simplified if one can suppose that the mappings $F$ of $C$ into $M$ are regular, that is, that the representation of the point $F(z)$ in terms of the parameter $\theta$ defining $z=e^{\theta i}$ has a form

$$
F(z)=\left[a_{1}(\theta), a_{2}(\theta), a_{3}(\theta)\right]
$$

in which $a_{i}(i=1,2,3)$ has a continuous derivature $\dot{a}_{i}$ and

$$
\dot{a}_{1}^{2}(\theta)+\dot{a}_{2}^{2}(\theta)+\dot{a}_{3}^{2}(\theta) \neq 0 .
$$

This and more is needed, and is supplied by the following lemma.

Lemma 5.2. Let e be a positive constant. Any $R$-invariant $L$-S-closed p-curve $F$ on $M$ admits an $R$-invariant $L$-S-deformation on $M$ into a p-curve $F^{\prime}$ on $M$ with no point $F(z)$ thereby displaced a distance more 
than e, and with $F^{\prime}$ regular.

The method of proof of this lemma is entirely similar to the methods used in proving Theorems 28.2 and 28.3 of Morse [2], except for the conditions of $R$-invariance of the $p$-curves used. Disregarding this condition for the moment recall that the component deformations used in Morse [2] are local in character, involving among other procedures the use of conformal transformations. All this is essentially the same on the sphere $M$. Short straight arcs used in the plane are here replaced by short geodesics on $M$. If the successive local deformations $D$ are applied to sufficiently restricted arcs $h$ on $M$, it will be possible to accompany each $D$ by a simultaneous deformation of the reflection $h^{\prime}$ of $h$ in the origin through a reflection of the deforms $h^{t}$ of $h$ under $D$. In this way the resultant deformations will be made $R$-invariant as required.

The order $Q\left(F_{1}, E\right)$. We shall refer to the given system of coordinates $\left(x_{1}, x_{2}, x_{3}\right)$ on $M$ as the system $E$. The points

$$
Z_{1}=(0,0,1), \quad Z_{-1}=(0,0,-1)
$$

will be called the poles of $E$. A p-curve or arc on $M$ whose carrier does not intersect the poles of $E$ will be termed $E$-pole free. A p-curve on $\Pi$ will be termed $E$-pole free if no point of its carrier is covered by a pole of $E$ on $M$. Let $F$ be an $R$-invariant closed $p$-curve of $M$ which is $E$-pole free. With $F(z)$ of the form

$$
F(z)=\left[x_{1}(z), x_{2}(z), x_{3}(z)\right]
$$

we set

$$
Q\left(F_{1}, E\right)=\underset{C_{1}}{\operatorname{variation}}\left[\frac{\operatorname{arc} x_{1}(z)+i x_{2}(z)}{\pi}\right]
$$

as $z$ traverses $C_{1}$ from $z=1$ to $z=-1$. Observe that $x_{1}(z)$ and $x_{2}(z)$ do not vanish simultaneously since $F$ is $E$-pole free. Thus $Q\left(F_{1}, E\right)$ is well defined. Moreover $Q\left(F_{1}, E\right)$ is an odd integer since

$$
x_{1}(-1)=-x_{1}(1), \quad x_{2}(-1)=-x_{2}(1) .
$$

We define $Q(F, E)$ similarly with $C_{1}$ replaced by $C$ in (5.5), and obobserve that $Q(F, E)=2 Q\left(F_{1}, E\right)$.

The following lemma is of the nature of a procedural simplification.

Lemma 5.3. Let $F$ be an $R$-invariant closed p-curve on $M$. The order $Q\left(F_{1}, E\right)$ is an invariant of any $R$-invariant deformation of $F$ on $M$ in which the deforms $F^{t}$ of $F$ remain $E$-pole free. The $R$-invariant $L-S$ homotopy class of $F$ contains p-curves $F^{*}$ such that 


$$
Q\left(F^{*}, E\right)=2 .
$$

The first affirmation of the lemma is immediately clear. In establishing the concluding statement of the lemma no generality will be lost if $F$ is assumed regular.

We shall deform an arc of $F_{1}$ over one of the poles of $E$. More definitely we start with an open simple arc $g$ of $F_{1}$ and deform the middle third $g_{1}$ of $g$, leaving the carrier of the residue of $g$ invariant in order that the deforms $g^{t}$ of $g$ may cause no failure of $F_{1}^{t}$ to remain L-S, apart from a failure of $g^{t}$ itself to remain L-S. We deform $g_{1}$ through tongue shaped curves $g_{1}^{b}$ with two end points fixed on $g$, and with semi-circular tips $\tau^{t}$. We suppose $\tau^{t}$ moves across $(0,0,-1)$ so that at the moment of crossing $(0,0,-1)$ is at the mid point of $\tau^{t}$. By virtue of such a crossing $Q\left(F_{1}^{t}, E\right)$ will change by 2 or -2 according as the sense of $\tau^{t}$ just after the moment $t_{0}$ of crossing is or is not the sense in which arc $\left(x_{1}+i x_{2}\right)$ increases on $\tau^{t}$. By an appropriate deformation in which the tongue remains L-S, either case can be made to happen. It should be observed that the tongue can be made selfintersecting provided it remains L-S. Since any finite number of such tongues can be used, it is clear that (5.6) can be made to hold provided the deformation of $F_{1}$ through the above $p$-arcs $F_{1}^{t}$ be converted into an $R$-invariant L-S deformation of $F$ by deforming the kernel residue $F_{2}$ of $F$ through $p$-arcs $F_{2}^{t}$ for which

$$
F_{2}^{t}(-z)=-F_{1}^{t}(z)
$$$$
\left[z \in C_{1}\right] .
$$

Canonical p-curves on $M$. These curves are special $p$-curves introduced to simplify the proof of Theorem 5.1. Such $p$-curves are to be regular $R$-invariant closed $p$-curves with the following properties:

(I) $F( \pm 1)=( \pm 1,0,0)$.

(II) The positive tangent to the path of $F$ at the points corresponding to $z= \pm 1$ on $C$ shall have the direction cosines $(0, \pm 1,0)$ respectively.

(III) The p-curve F shall be E-pole free.

(IV) The order $Q(F, E)=2$.

It follows from Lemmas 5.2 and 5.3 that there exists a canonical $p$-curve $F$ in the $R$-invariant L-S-homotopy class of any given $R$-invariant L-S-p-curve. Cf. proof of Lemma 7.1. We term a kernel $F_{1}$ of a canonical $p$-curve $F$, a canonical kernel $F_{1}$. Canonical kernels lie on the open sub-manifold of $M$

$$
M_{1}=M-Z_{1}-Z_{-1} \quad\left[Z_{ \pm 1}=(0,0, \pm 1)\right] .
$$

We shall make several uses of the following mapping. 
$A$ mapping $W$ of $M_{1}$ into a complex w-plane. Under this mapping $x$ in $M_{1}$ has an image $w=W(x)$ in the $w$-plane where

$$
W(x)=\exp \left[x_{3}+2 i \operatorname{arc}\left(x_{1}+i x_{2}\right)\right] \quad\left(x \in M_{1}\right) \text {. }
$$

This mapping can be equivalently given in the form

$$
|w|=\exp \left[x_{3}\right], \quad \operatorname{arc} w=2 \operatorname{arc}\left(x_{1}+i x_{2}\right) .
$$

The mapping $W$ is single-valued and continuous, and carries $M_{1}$ into a ring in the w-plane on which

$$
e^{-1}<|u|<e .
$$

Each point $w$ in this ring has just two distinct points on $M_{1}$ of the form

$$
\left.\left(a_{1}, a_{2}, a_{3}\right) \quad\left(-a_{1},-a_{2}, a_{3}\right) \quad\left[\left(a_{1}, a_{2}\right) \neq(0,0)\right]\right\}
$$

as antecedents. The mapping $W$ is locally top. The inverse $W^{-1}$ is single-valued on a two-sheeted Riemann surface covering the ring (5.10) twice without branch points.

Canonical p-curves in the w-plane. If $F_{1}$ is a canonical kernel on $M$, there exists a unique regular, closed $p$-curve $\Omega$ mapping the circle $C$ into the ring (5.10) on the w-plane, and such that

$$
\Omega(z)=W F_{1} \mu(z) \quad[z \in C] .
$$

Such a $p$-curve has the following properties, paralleling the properties I-IV of canonical $p$-curves on $M$.

(I') $\Omega(1)=1$.

(II') The positive tangent to the path of $\Omega$ at the point corresponding to $z=1$ is parallel to the positive $v$-axis $(u+i v=w)$.

(III') The carrier of $\Omega$ is on the ring (5.10).

(IV') The ordinary plane order of $\Omega$ with respect to $w=0$ is 1 .

Conversely any closed regular $p$-curve $\Omega$ in the w-plane which is canonical in the above sense determines a unique canonical kernel $F_{1}$ on $M$ such that (5.11) holds. We then term $F$ the $\mu$-antecedent on $M$ of $\Omega$ in the w-plane. Any L-S-deformation of a canonical kernel on $M_{1}$ or $p$-curve on the ring (5.10) through such curves will be called canonical.

Let $\Omega$ then be a canonical $p$-curve $\Omega$ on the $\operatorname{ring}(5.10)$ and $F$ its canonical $\mu$-antecedent on $M$. Any canonical L-S-deformation $\Omega^{t}, 0 \leqq t \leqq 1$, of $\Omega$ on the ring (5.10) implies a canonical L-S-deformation $F_{1}^{t}, 0 \leqq t \leqq 1$, of $F_{1}$ on $M$ such that

$$
\Omega^{t}=W F_{1}^{t} \mu
$$


Such deformations of $\Omega$ on the ring (5.10) are 0 -deformations in the sense of Theorem 33.1 of Morse [2]. Since the ordinary plane order $q$ of $\Omega$ is 1 the proof ${ }^{4}$ of Lemma 33.1 and Theorem 33.1 shows that $\Omega$ admits a canonical L-S-deformation on the ring (5.10) into a $p$-curve $\Omega^{1}$ whose $\mu$-antecedent on $M$ has the kernel $\{\gamma\}$ or $\left\{\gamma \lambda^{p-1}\right\}$ according as the angular order (cf. Morse [2]) $p$ of $\Omega$ is 1 or not 1 . Lemma 5.1 thus permits the following conclusion.

Lemma 5.4. A canonical kernel $F_{1}$ on $M$ admits a canonical L-S-deformation on $M$ into $\{\gamma\}$ or $\{\gamma \lambda\}$.

Observe that $\{\gamma\}$ is the kernel $\Gamma_{1}$ of the $R$-invariant $p$-curve $\Gamma$ on $M$ defined at the end of $\S 4$. Recall that $A \Gamma \mu=k^{(1)}$. Observe further that the circle $\lambda$ can be deformed through circles which remain tangent to $\gamma$ at $(0,1,0)$ into a great circle $C^{\prime}$ on which $x_{3}=0$, so that $\{\gamma \lambda\}$ is L-S-deformable among canonical kernels into $\left\{\gamma C^{\prime}\right\}$. This is the kernel of $\Gamma^{(3)}$. Recall that $A \Gamma^{(3)} \mu=k^{(3)}$. We are thus led to the basic theorem.

Theorem 5.1. Any L-S-closed p-curve $f$ not $\approx 0$ on $\Pi$ is in the L-Shomotopy class of $k^{(1)}$ or $k^{(3)}$.

We have merely to review the various steps which lead to this result. In the first place the given $f$ has an $R$-invariant closed $p$-curve $F$ as a $\mu$-antecedent on $M$. Cf. Lemma 4.2. Such an $F$ admits an $R$-invariant L-S-deformation into a canonical $p$-curve $F^{*}$. Cf. Lemmas 5.2 and 5.3, and the proof of Lemma 7.1.

The kernel $F_{1}^{*}$ admits a L-S-deformation through canonical kernels $F_{1}^{* t}$ into $\{\gamma\}$ or $\{\gamma \lambda\}$ in accordance with Lemma 5.4, and hence into the canonical kernel of $\Gamma$ or $\Gamma^{(3)}$. On extending these canonical kernels on $M$ by reflection as in (5.7) we infer that $F^{*}$ admits a L-S-deformation on $M$ through $R$-invariant $p$-curves $F^{* t}$ into $\Gamma$ or $\Gamma^{(3)}$. The p-curves $A F_{1}^{* t} \mu$ on $I I$ are closed and L-S, and deform $A F_{1}^{*} \mu$ into $k^{(1)}$ or $k^{(3)}$. In résumé, $f=A F_{1} \mu$ is first L-S-deformed on $\Pi$ into $A F_{1}^{*} \mu$ and then into $k^{(1)}$ or $k^{(3)}$.

This completes the proof of the theorem.

It remains to show that $k^{(1)}, k^{(2)}, k^{(3)}, k^{(4)}$ are in distinct L-Shomotopy classes on $\Pi$. Part of this result is already clear. For the property of a $p$-curve $f$ being null homotop. on $\Pi$ is invariant of arbitrary continuous deformations of $f$ on $\Pi$ and in particular invariant of L-S-deformations. Thus the null homotop. p-curves $k^{(2)}$ and $k^{(4)}$

${ }^{4}$ In the proof of Lemma 33.1 suppose that a line element $E$ of $g$ at $Q$ is tangent to a circle $C$ with center at $w=0$. One can hold $E$ fast in the deformation. A preliminary L-S-deformation should be used to make $g$ convex towards the origin near $Q$. One then proceeds as before identifying $Q$ with the point $s=a$ of the proof. 
are not in the L-S-homotopy classes of $k^{(1)}$ and $k^{(3)}$. Moreover $\left[k^{(2)}\right] \neq\left[k^{(4)}\right]$ as affirmed in Theorem 4.2. We must finally show that

$$
\left[k^{(3)}\right] \neq\left[k^{(1)}\right]
$$

and going somewhat deeper characterize the classes $\left[k^{(3)}\right]$ and $\left[k^{(1)}\right]$ topologically.

In $\$ 6$ a numeral invariant $d(f, E)$ of a L-S-homotopy class $[f]$ is defined in case $f$ not $\approx 0$ and $f$ is $E$-pole free. In $\S 7, d(f, E)$ is replaced by a topological invariant $d_{S}(f)$ defined for an arbitrary top. image $S$ of the projective plane, thereby freeing $d(f, E)$ from its dependence on the special coordinate system $E$ and the special representation $\Pi$ of a projective plane.

6. The difference order $d(f, E)$ when $f$ not $\approx 0$. Let $F$ be an $R$-invariant closed $p$-curve on $M$. In the case in which $F$ is $E$-pole free an angular order $P\left(F_{1}, E\right)$ of the kernel $F_{1}$ will be defined. For this purpose it is necessary that $M$ receive an orientation from $E$.

The E-orientation of $M$. Corresponding to the coordinate system $E$ of $M, M$ will be oriented as follows. Let $C(x)$ be an arbitrarily small circle on $M$ with center at $x$ in $M$. As previously, let $Z_{ \pm 1}=(0,0, \pm 1)$. The positive sense of $C\left(Z_{-1}\right)$ shall be such that a continuous branch of the multiple-valued function

$$
\operatorname{arc}\left(y_{1}+i y_{2}\right) \quad\left[y=\left(y_{1}, y_{2}, y_{3}\right)\right]
$$

increases as $y$ traces $C\left(Z_{-1}\right)$ in its positive sense. The sense of $C(x)$ at other points $x$ in $M$ will be obtained by a continuous variation of $C(x)$ from $C\left(Z_{-1}\right)$. In particular it should be noted that as $C\left(Z_{1}\right)$ is traced in its positive sense by a point $y$ any continuous branch of arc $\left(y_{1}+i y_{2}\right)$ decreases.

Reference directions for the measurement of angles at a point $x$ of $M_{1}=M-Z_{1}-Z_{-1}$ must be defined. For each $x$ in $M_{1}$ let $C^{1}(x)$ be the circle through $x$ parallel to the plane on which $x_{3}=0$. Let the positive sense of $C^{1}(x)$ be that of increasing arc $\left(y_{1}+i y_{2}\right)$ for $y$ in $C^{1}(x)$. The reference direction at $x$ shall be the positive tangent to $C^{1}(x)$ at $x$. The sign of an angle at $x$ measured from the reference direction will be determined by the orientation of $M$ at $x$ as defined by $C(x)$.

The angular order $P\left(F_{1}, E\right)$. Let $F$ be $R$-invariant L-S, closed and $E$-pole free. Set $f=A F_{1} \mu$. Let $\epsilon_{1}$ be a positive constant so small that the submappings of $F$ on which $z=e^{i \theta}$ with $\alpha \leqq \theta \leqq \alpha+\epsilon_{1}$ are top. mappings for each constant $\alpha$. Given $z=e^{i \theta}$ let $z_{\epsilon}$ denote the point $e^{(\theta+\epsilon) i}$. We suppose that $0<\epsilon<\epsilon_{1}$. Let $H_{F}(z, \epsilon)$ denote the angle at the point $x=F(z)$ in $M$, measured from the reference direction at $x$ to the positive tangent at $x$ to the great circle on $M$ leading from $F(z)$ to 
$F\left(z_{\epsilon}\right)$. For fixed $\epsilon$ let $H_{F}(z, \epsilon)$ be chosen so as to vary continuously with $z$ in $\bar{C}_{1}$. Since $F(-z)=-F(z)$ it is clear that

$$
H_{F}(-1, \epsilon)=-H_{F}(1, \epsilon)
$$

so that

$$
H_{F}(-1, \epsilon)+H_{F}(1, \epsilon)=2 r \pi
$$

where $r$ is an integer. We set

$$
P\left(F_{1}, E\right)=\frac{H_{F}(-1, \epsilon)+H_{F}(1, \epsilon)}{\pi}
$$

and note the following.

The value of $P\left(F_{1}, E\right)$ is independent $\bmod 4$ of the choice of $\epsilon$ in $\left(0, \epsilon_{1}\right)$, of the choice of $F$ between the two $\mu$-antecedents of $f$, and of the choice of $H_{F}$ among the possible continuous branches of this angle function. For $H_{F}(z, \epsilon)$ can be chosen as to vary continuously with $(z, \epsilon)$ for $\epsilon$ in $\left(0, \epsilon_{1}\right)$ and $z$ in $\bar{C}_{1}$, so that the left member of (6.2) is independent of $\epsilon$ in $\left(0, \epsilon_{1}\right)$. If $F$ and $F^{*}$ are the two $\mu$-antecedents of $f, F(z)=-F^{*}(z)$, so that one can take

$$
H_{F^{*}}(z, \epsilon)=-H_{F}(z, \epsilon) \text {. }
$$

Hence

$$
P\left(F_{1}^{*}, E\right)=-P\left(F_{1}, E\right)=P\left(F_{1}, E\right)
$$

Finally a change of the continuous branch of $H_{F}$ will change the left member of (6.2) by an integral multiple of $4 \pi$ and so leave $P\left(F_{1}, E\right)$ unchanged mod 4.

The difference order $d(f, E)$. Let $f$ not $\approx 0$ be a L-S-closed $p$-curve on $I I$ which is $E$-pole free. Let $F$ be a $\mu$-antecedent on $M$ of $f$ on $\Pi$. We set

$$
d(f, E) \equiv Q\left(F_{1}, E\right)-P\left(F_{1}, E\right)
$$

and observe that $d(f, E)$ is independent of the choice of $F$ as $\mu$-antecedent of $f$, and of any $R$-invarient L-S-deformation of $F$ on $M_{1}$.

One sees that

$$
\begin{array}{ll}
d\left(k^{(1)}, E\right) \equiv Q\left(\Gamma_{1}, E\right)-P\left(\Gamma_{1}, E\right)=1-0 & (\bmod 4), \\
d\left(k^{(3)}, E\right) \equiv Q\left(\Gamma_{1}^{(3)}, E\right)-P\left(\Gamma_{1}^{(3)}, E\right)=3-0 & (\bmod 4) .
\end{array}
$$

An immediate conclusion is that $k^{(1)}$ admits no L-S-deformation on $\Pi$ into $k^{(3)}$ through $p$-curves which are $E$-pole free. To remove the latter condition, deformations must be made through the poles of $E$ and 
the effect on $d(f, E)$ determined. For this purpose $p$-curves on $\Pi$ whose $\mu$-antecedents on $M$ are broken geodesics are useful.

Admissible broken geodesics on $M$. In Morse and Heins [1] use has been made of L-S-curves composed of sequences of a finite number of straight arcs. The analogous $p$-curves on $M$ are sequences of a finite number of geodesic arcs each less than $\pi$ in length, with nonzero angles at the vertices (the junction points of successive arcs). A p-curve on $M$ of this character will be called an admissible broken geodesic. Admissible broken geodesics are L-S. A deformation on $M$ of an $R$-invariant closed $p$-curve $F$ through admissible broken geodesics $F^{t}$ will be termed admissible if the number of vertices is independent of $t$, if the vertices vary continuously with $t$ and remain distinct on any $p$-curve $F^{t}$, if the point $F^{t}(1)$ is a vertex of $F^{t}$, and if each $p$-curve $F^{t}$ is $R$-invariant. The methods of Morse and Heins [1] suffice to prove the following lemma.

Lemma 6.1. Let e be a positive constant. Any $R$-invariant $L-S$-p-curve $F$ on $M$ admits an $R$-invariant $L$-S-deformation into an admissible broken geodesic displacing each point $F(z)$ on $M$ at most e in this process.

Any two $R$-invariant broken geodesic closed $p$-curves $F$ and $F^{\prime}$ which are in the same $R$-invariant L-S-homotopy class, can be admissibly deformed on $M$ into each other through $R$-invariant broken geodesics, provided a suitable number of vertices are initially added to $F$ and $F^{\prime}$.

With this lemma as an aid, the following theorem can be proved:

THEOREM 6.1. If $f$ not $\approx 0$ and $f^{\prime}$ not $\approx 0$ are two closed $L-S$ - $p$-curves on $\Pi$ in the same homotopy class on $\Pi$ and if $f$ and $f^{\prime}$ are E-pole free, then

$$
d(f, E)=d\left(f^{\prime}, E\right)
$$

The theorem follows at once from the definition of $d(f, E)$ if $f$ can be L-S-deformed into $f^{\prime}$ on II through $p$-curves which are $E$-pole free. In any other case we can suppose, without loss of generality, that the $\mu$-antecedents $F$ and $F^{\prime}$ on $M$, of $f$ and $f^{\prime}$ respectively on $\Pi$, are admissible broken geodesics which are $E$-pole free. In accordance with Lemma 6.1, $F$ can be admissibly deformed into $F^{\prime}$ through a family $F^{t}$ of broken geodesics. If use is made of the freedom of small displacements of the vertices of $F^{t}$, we can be assured that $F^{t}$ is $E$-pole free except for a finite set of values $t_{1}, \cdots, t_{n}$ of $t$, that no vertex of $F^{t_{i}}(i=1, \cdots, n)$ is at a pole of $E$, and that $F^{t_{i}}$ has just one point in common with the poles of $E$.

The conventions as to the measurement of angles are such that as a geodesic arc of $F_{1}^{t}$ moves across the pole $(0,0,1)$ 


$$
\Delta P\left(F_{1}^{t}, E\right)=\Delta Q\left(F_{1}^{t}, E\right)= \pm 2
$$

so that $d\left(f^{t}, E\right)$ is unchanged by such a passage. When a geodesic arc of $F_{1}^{t}$ moves across the pole $(0,0,-1)$

$$
\Delta P\left(F_{1}^{t}, E\right)=-\Delta Q\left(F_{1}^{t}, E\right)= \pm 2 .
$$

The difference $d\left(f^{t}, E\right) \bmod 4$ is accordingly invariant as $t$ increases from 0 to 1 . This completes the proof of the theorem.

Reference to (6.4) gives the following corollary of the theorem.

COROLlARY 6.1. The models $k^{(1)}$ and $k^{(3)}$ on $\Pi$ are not in the same L-S-homotopy class on $\Pi$.

By virtue of Theorems 5.1 and 6.1 any L-S-p-curve $f$ not $\approx 0$ on $\Pi$ which is $E$-pole free is in the L-S-homotopy class of $k^{(1)}$ or $k^{(3)}$ according as $d(f, E)=1$ or $3 \bmod 4$. This determination of the L-S-homotopy class of $f$ depends upon our special model $\Pi$ of the projective plane and upon the coordinate system $E$. We shall remove this dependency.

7. Invariant orders and models. We begin with the following lemma:

LemMa 7.1. Any simple closed p-curve $f$ not $\approx 0$ on $\Pi$ II can be deformed on $\Pi$ into any other such p-curve on $\Pi$ through simple, closed p-curves.

It will be sufficient to show that $f$ can be deformed into $k^{(1)}$ in the manner required. If $F$ is a $\mu$-antecedent of $f$ it will be sufficient to show that $F$ can be deformed on $M$ into $\Gamma$ through $R$-invariant simple, closed $p$-curves on $M$. The required deformation will be defined as a sequence of five deformations.

(1) We first deform $F$ in the required manner into an $R$-invariant, simple, regular closed $p$-curve $F^{(1)}$. With obvious precautions to maintain a simple curve the proof of Lemma 5.2 will suffice.

(2) We next rotate $M$ in such a manner that $F^{(1)}$ is deformed into a p-curve $F^{(2)}$ for which $F^{(2)}(1)=1$.

(3) A suitable rotation of $M$ about the $x_{1}$ axis will then carry $F^{(2)}$ into a $p$-curve $F^{(3)}$ which is tangent to $\Gamma$ at the point $(1,0,0)$.

(4) If $F_{1}^{(3)}$ is not $E$-pole free its kernel $F_{1}^{(3)}$, with its simple projection on $\Pi$, intersects $(0,0,1)$ in a point $F_{1}^{(3)}(z)$ for just one value of $z$ on $C_{1}$. A suitable L-S-deformation of $F_{1}^{(3)}$ near this point of intersection and a corresponding $R$-invariant L-S-deformation of $F^{(3)}$ will yield a simple closed $p$-curve $F^{(4)}$ which is $E$-pole free. Moreover

$$
Q\left(F^{(4)}, E\right)= \pm 2
$$

as one sees on projecting $M_{1}$ stereographically from $(0,0,1)$ onto the 
plane tangent to $M$ at $(0,0,-1)$. Finally we can suppose that the + sign holds in (7.0). For if one keeps $F_{1}^{(4)}$ simple and regular and deforms a tongue once over $(0,0,1)$, the order $(7.0)$ of $F^{(4)}$ will be changed from -2 to 2 , if initially -2 .

(5) The resultant $p$-curve $F^{(4)}$ is canonical in the sense of $\$ 5$. Use can be made of the mapping $W$ of $M_{1}$ into the ring (5.10) of the wplane. On this ring there exists a canonical $p$-curve $\Omega$ such that

$$
\Omega=W F_{1}^{(4)} \mu \text {. }
$$

In particular $\Omega$ has the plane order 1 relative to $w=0$ in the $w$-plane. It follows that there exists a deformation of $\Omega$ on the ring (5.10) through simple, canonical $p$-curves $\Omega^{t}, 0 \leqq t \leqq 1$, on the ring into the $p$-curve $w=z=e^{\theta i}$. On $M, F$ can accordingly be L-S-deformed through simple canonical $p$-curves into $\Gamma$.

Hence $f$ can be deformed on $\Pi$ in the manner required into $k^{(1)}$.

Various methods (including conformal mapping) are available to prove the following.

(i) Let $g^{t}, 0 \leqq t \leqq 1$, be a 1 -parameter family of simple closed $p$-curves in the $(u, v)$-plane of which $g^{1}$ is the circle $C: z=e^{i \theta}$. Let $G^{t}$ be the closure of the interior of $g^{t}$. There exists a continuous 1 -parameter family of top. mappings $T^{t}$ of $G^{t}$ into the closed disc bounded by $C$, such that $T^{t}$ maps $g^{t}(z)$ into $z$ and $G^{1}$ is the identity.

Recall that an isotopic deformation of a manifold $S$ is defined by a continuous 1-parameter family of top. mappings of $S$ onto $S$. With this understood we state the following lemma. In proving this lemma it will be convenient to denote the carrier of a $p$-curve $F$ by $|F|$.

Lemma 7.2. Any homeomorphism $H$ of $\Pi$ can be isotopically deformed into the identity on $\mathrm{II}$.

Let $K$ be an $R$-invariant homeomorphism of $M$ such that $A K=H$. Set $F=K^{-1} \Gamma$. By virtue of Lemma 7.1 there exists a continuous 1 -parameter family of $R$-invariant, simple, closed $p$-curves $F^{t}$ on $M$ which deform $F$ into $\Gamma$. Let $\Sigma^{t}, 0 \leqq t \leqq 1$, be a continuous 1 -parameter family of closed domains on $M$ bounded by the respective Jordan curves $\left|F^{t}\right|$. Observe that $\Sigma^{1}$ is a hemisphere of $M$ bounded by $|\Gamma|$. It follows from (i) that there exists a continuous 1-parameter family of top. mappings $T^{t}$ of $\Sigma^{t}$ onto the hemisphere $\Sigma^{1}$, which, in particular, map the Jordan curve $\left|F^{t}\right|$ onto the circle $|\Gamma|$ in such a manner that $\Gamma(z)$ is the image of $F^{t}(z)$ and $T^{1}$ is the identity. The mappings $T^{t}$ can be extended over $M$ by reflection, that is, so that

$$
T^{t}(-x)=-T^{t}(x) \quad(X, t) \in(M \times J) .
$$


So extended $T^{t}, 0 \leqq t \leqq 1$, defines an isotopic deformation of $T^{0}$ into the identity $T^{\mathbf{1}}$.

It remains to deform $K$ isotopically into $T^{0}$. By definition $K F=\Gamma$ so that $K(x)=T^{0}(x)$ when $x$ is in the Jordan curve $|F|$. By a theorem of Tietsze there is an isotopic deformation of the mapping $K$ restricted to $\Sigma^{0}$, into $T^{0}$, likewise restricted to $\Sigma^{0}$, leaving $|F|$ pointwise fixed. This deformation can be extended to all of $K$ by reflection in the origin, so as to yield an $R$-invariant isotopic deformation of $K$ into $T^{0}$. Hence $K$ is isotopically deformable in to the identity through $R$-invariant top. mappings of $M$ onto $M$.

The lemma follows.

Proof of Theorem 1.1 of the introduction. The $p$-curve $k$ whose multiple tracings $k^{(1)}, k^{(2)}, k^{(3)}, k^{(4)}$ appear in Theorems 4.2 and 5.1 is a simple, closed $p$-curve on $\Pi$ with $k$ not $\approx 0$ on $\Pi$. It follows from Lemma 7.1 that in these theorems $k$ can be replaced by any other simple, closed $p$-curve $h$ such that $h$ not $\approx 0$. This completes the proof of the fundamental Theorem 1.1.

Further invariance of $d(f, E)$. We now admit any coordinate system $E^{\prime}$ obtained from $E$ by a rotation of $E$ about the origin, or by a reflection of $E^{\prime}$ in the origin. We have seen that $d(f, E)$ is independent of the L-S-deformation class of $f$ provided only that $d(f, E)$ is well defined, that is, provided that $f$ is $E$-pole free. The following theorem shows the essential top. invariance of $d(f, E)$.

THEOREM 7.1. Let $f$ be a L-S-closed $p$-curve on $\Pi, H$ a homeomorphism of $\Pi$ and $f^{\prime}=H f$ the transform of $f$ under $H$. If $E$ and $E^{\prime}$ are admissible coordinate systems such that $f$ and $f^{\prime}$ are respectively $E$ and $E^{\prime}$-pole free, then

$$
d(f, E)=d\left(f^{\prime}, E^{\prime}\right) .
$$

We shall first show that

$$
d(f, E)=d(H f, E)
$$

provided $f$ and $H f$ are $E$-pole free. Relation (7.2) follows from Lemma 7.2 according to which $H$ can be isotopically deformed into the identity, thus implying a L-S-deformation of $H f$ into $f$. From the invariance of $d(f, E)$ under such deformations of $f,(7.2)$ must hold.

We shall next show that

$$
d(f, E)=d\left(f, E^{\prime}\right)
$$

provided $f$ is $E$ and $E^{\prime}$-pole free. To that end let $T$ be the orthogonal transformation by virtue of which $E^{\prime}=T E$. It is trivial that 


$$
d(f, E)=d(T f, T E) .
$$

But $f$ and $f^{\prime}$ are $E^{\prime}$-pole free so that

$$
d(T f, T E)=d(f, T E)=d\left(f, E^{\prime}\right)
$$

according to (7.2). Hence (7.3) holds.

To establish (7.1) let $E^{\prime \prime}$ be chosen (as is possible) so that $f$ and $f^{\prime}$ are $E^{\prime \prime}$-pole free. By hypothesis $f$ is $E$-pole free, and $f^{\prime}$ is $E^{\prime}$-pole free. Hence

$$
d(f, E)=d\left(f, E^{\prime \prime}\right)=d\left(f^{\prime}, E^{\prime \prime}\right)=d\left(f^{\prime}, E^{\prime}\right)
$$

in accordance with (7.2) and (7.3). This completes the proof of the theorem.

Definition of an invariant $S$-order of $f$ when $f$ not $\approx 0$. Let $S$ be an arbitrary top. model of the projective plane, and $f$ an L-S-closed $p$ curve on $S$ with $f$ not $\approx 0$ on $S$. Then $d(Z f, E)$ is independent mod 4 of the choice of $Z$ among top. mappings of $S$ onto $\Pi$ and of the choice of $E$ among admissible rectangular coordinate systems for $M$ provided $Z f$ is $E$-pole free.

For each $L$-S-closed p-curve of not $\approx 0$ on $S$ we set

$$
d(Z f, E)=d_{S}(f)
$$

provided $Z f$ is $E$-pole free, and term $d_{S}(f)$ the $S$-difference order of $f$.

The fundamental nature of the top. invariance of $d_{S}(f)$ is specified in the following theorem.

THEOREM 7.2. The $S$-difference order $d_{S}(f)$ of a L-S-closed p-curve $f$ not $\approx 0$ on $S$ is independent of the choice of $f$ in its L-S-homotopy class. If $S$ is mapped top. onto $S^{\prime}$ under a mapping $K$ and if $f^{\prime}=K f$, then

$$
d_{S}(f)=d_{S^{\prime}}\left(f^{\prime}\right)
$$

The difference order $d_{S}(f)$ has but two possible values 1 and 3 , mod 4 . $A$ necessary and sufficient condition that $d_{S}(f)=1 \bmod 4$ is that the $L$-S-homotopy class of $f$ contain a simple, closed p-curve $f_{1}$ not $\approx 0$ on $S$.

Let $Z$ and $Z^{\prime}$ be arbitrary top. mappings of $S$ and $S^{\prime}$ respectively onto $I$. Then by definition

$$
d_{S}(f)=d(Z f, E), \quad d_{S^{\prime}}\left(f^{\prime}\right)=d\left(Z^{\prime} f^{\prime}, E^{\prime}\right)
$$

provided $E$ and $E^{\prime}$ are admissible coordinate systems for $M$ such that $Z f$ and $Z^{\prime} f^{\prime}$ are respectively $E$ and $E^{\prime}$-pole free. Observe that

$$
Z^{\prime} f^{\prime}=\left(Z^{\prime} K Z^{-1}\right)(Z f)
$$


and that the transformation

$$
Z^{\prime} K Z^{-1}=H
$$

is a top. mapping of $\Pi$ onto $\Pi$. Hence

$$
d(Z f, E)=d\left(Z^{\prime} f^{\prime}, E^{\prime}\right)
$$

in accordance with (7.1). Thus (7.5) holds.

The first statement in the theorem is a consequence of Theorem 6.1 .

To establish the last statement in the theorem suppose first that $d_{S}(f)=1$. Recall that $[f]=\left[k^{(1)}\right]$ or $\left[k^{(3)}\right]$ by Theorem 5.1 . But we have seen in (6.4) that

$$
d\left(k^{(1)}, E\right)=1, \quad d\left(k^{(3)}, E\right)=3
$$

so that $[f]=\left[k^{(1)}\right]$. Thus $k^{(1)}$ is a simple closed $p$-curve in $[f]$ as affirmed.

Conversely, suppose that $[f]$ contains a simple, closed $p$-curve $f_{1}$. Recall that $\left[f_{1}\right]=\left[k^{(1)}\right]$ as a consequence of Lemma 7.1. Hence

$$
d_{S}(f)=d\left(k^{(1)}, E\right)=1
$$

$(\bmod 4)$.

This completes the proof of the theorem.

The part of Theorem 1.1 which concerns the case $f$ not $\approx 0$ can be completed as follows.

THEOREM 7.3. If $f$ not $\approx 0$ is a L-S-closed p-curve on the top. image $S$ of a projective plane $[f]=\left[k^{(1)}\right]$ or $\left[k^{(3)}\right]$ according as $d_{S}(f)=1$ or $3 \bmod 4$.

\section{HEINZ HopF}

\section{REFERENCES}

Ueber die Drehung der Tangenten und Lehnen ebener Kurven, Compositio Math. vol. 2 (1935) pp. 50-62.

H. WHITNEY

On regular closed curves in the plane, Compositio Math. vol. 4 (1937) pp. 276-284. M. MORSE

1. L-S-homotopy classes of locally simple curves, Annales de la Société Polonaise de Mathématique vol. 21 (1948) pp. 236-256.

2. Topological methods in the theory of functions of a complex variable, Princeton University Press, 1947.

M. MoRSE AND M. Heins

1. Topological methods in the theory of functions of a single complex variable, I. Deformation types of locally simple curves, Ann. of Math. vol. 46 (1945) pp. 600-624.

2. Deformation classes of meromorphic functions and their extensions to interior transformations, Acta Math. vol. 79 (1946) pp. 51-103.

INSTITUTE FOR ADVANCEd STUdy 